\title{
C-B- 样条曲线的升阶算子与几何生成法
}

\author{
朱平(1)(2)，汪国昭 ${ }^{(1)} ，$ 于静静 ${ }^{(1 *}$ \\ (1) 浙江大学数学系计算机图像图形研究所, 杭州 310027 \\ (2) 东南大学科学计算实验室, 南京 211189 \\ *通信作者. E-mail: zhuping.zju@gmail.com,wanggz@zju.edu.cn, yjj82325@sohu.com \\ 收稿日期: 2008-08-25; 接受日期: 2009-04-03 \\ 国家自然科学基金 (批准号: 60773179，60970079) 和国家自然科学基金青年基金 (批准号: 60904070) 资助项目
}

摘要 因为升阶算子与几何收敛性不易求得, 故样条曲线很难像 Bézier 曲线那样 通过对控制多边形的几何割角生成. 为了得到 C-B-样条的几何生成算法, 首先利 用双阶样条解决了升阶算子. 接着, 证明了对样条曲线进行基于升阶算子的升阶, 其控制多边形序列会逐渐收敛到初始的样条曲线。这种几何生成算法具有明显的 几何直观性, 计算简单稳定, 利于硬件执行. 由于椭圆、螺旋线等工程上应用广泛 的曲线可以由 C-B-样条精确表示, 因此算法对 CAD 造型系统有重要的意义.

关键词

升阶算子

双阶 C-B-样条

几何收敛性

几何生成法

\section{1 引言}

近些年来, 为了克服 B-样条在几何设计中的缺点, 人们开发了许多新的样条模型. C-B-样条最初 就是构造在空间 $\{1, t, \sin t, \cos t\}^{[1,2]}$ 上的 3 次均匀样条. 后来 Wang 等 ${ }^{[3]}$ 将它扩展到 $n$ 次非均匀的情 况, 形成 NUAT(non-uniform algebraic-trigonometric) B-样条. C-B-样条不仅与多项式 B-样条拥有相同 的性质, 而且具有最佳保形性 ${ }^{[4]}$, 能够精确表示椭圆、螺旋线 ${ }^{[5]}$ 等曲线并克服了 NURBS(non-uniform rational B-spline) 的积分困难. 当它们被用来构造旋转曲面 ${ }^{[6]}$ 时, 得到的算法更简单, 更节省空间并

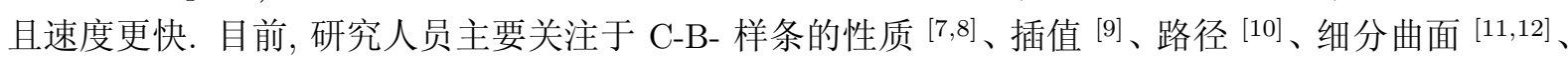
造型系统 ${ }^{[13]}$ 、形状参数 ${ }^{[14]}$ 以及高阶的精确表示 ${ }^{[15]}$. 总之, 对 C-B-样条的研究对造型系统具有重要 意义.

众所周知, Bézier 曲线可以通过对控制多边形的割角来生成 ${ }^{[16]}$. 这种生成算法几何直观性强, 计 算简单稳定, 十分有利于硬件实现. 但是对样条曲线而言, 由于升阶算子及相应的几何收敛性不易求 得, 所以样条曲线无法几何生成. 在 B- 样条曲线的升阶工作中, 鉴于以往的算法 ${ }^{[17 \sim 19]}$ 缺乏几何意 义 ${ }^{[20,21]}$, Wang 等 ${ }^{[22]}$ 在 2007 年提出双阶 B- 样条的概念, 使得 B- 样条的升阶有了几何意义. 这就为 C-B- 样条曲线的几何生成提供了可能.

在这篇文章中, 我们首先利用双阶样条的方法求得 C-B 样条的升阶算子, 并将这种升阶算法与以 往的算法做一比较. 然后利用 C-Bézier 基的性质得到了样条曲线的几何收玫性. 最后描述了 C-B- 样 条曲线的几何生成算法. 由于椭圆、螺旋线等工程上广泛应用的曲线都能用 C-B- 样条精确表示, 因此 这种生成算法对 CAD 造型系统具有重要意义. 


\section{C-B-样条曲线与升阶算子}

\subsection{C-B-样条基}

定义 $\mathbf{1}^{[3]}$ 令 $\boldsymbol{T}$ 是一给定的节点序列 $\left\{t_{i}, i=0, \pm 1, \ldots\right\}$ 并满足 $0 \leqslant t_{i+1}-t_{i} \leqslant \pi$. 对 $t \in\left[t_{i}, t_{i+k}\right]$, 由 下列公式构造的 $N_{i, k}(t)(k \geqslant 3)$ 称为在空间 $\left\{1, t, \ldots, t^{k-3}, \sin t, \cos t\right\}$ 上构造的 C-B- 样条基.

$$
N_{i, 2}(t)= \begin{cases}\frac{\sin \left(t-t_{i}\right)}{\sin \left(t_{i+1}-t_{i}\right)}, & \text { 当 } t_{i} \leqslant t<t_{i+1} ; \\ \frac{\sin \left(t_{i+2}-t\right)}{\sin \left(t_{i+2}-t_{i+1}\right)}, & \text { 当 } t_{i+1} \leqslant t<t_{i+2} ; \\ 0, & \text { 其他. }\end{cases}
$$

对 $k \geqslant 3, N_{i, k}(t)$ 由递归定义得到 $N_{i, k}(t)=\int_{-\infty}^{t}\left[\delta_{i, k-1} N_{i, k-1}(s)-\delta_{i+1, k-1} N_{i+1, k-1}(s)\right] \mathrm{d} s$, 这里 $\delta_{i, k}=\left(\int_{-\infty}^{+\infty} N_{i, k}(t) \mathrm{d} t\right)^{-1}, i=0, \pm 1, \ldots$

\subsection{C-B-样条曲线}

定义 $\mathbf{2}^{[3]}$ 一条 $k$ 阶 C-B-样条曲线定义如下: $\boldsymbol{P}(t)=\sum_{i=0}^{m} N_{i, k}(t) \cdot \boldsymbol{P}_{i}, t_{k-1} \leqslant t \leqslant t_{m+1}$, 这里 $\boldsymbol{P}_{i}$ $(i=0, \ldots, m)$ 是控制顶点, $\left\{N_{i, k}(t)\right\}_{i=0}^{m}$ 是对应到参数轴 $\boldsymbol{T}=\left\{t_{j}\right\}(j=0, \pm 1, \ldots)$ 上的 $k$ 阶 C-B-样条 基.

\section{3 升阶算子}

现在我们考虑 C-B-样条曲线的升阶算子. 一条 $k$ 阶 C-B-样条曲线: $\boldsymbol{P}(t)=\sum_{i=0}^{m} N_{i, k}(t) \cdot \boldsymbol{P}_{i}, N_{i, k}(t)$ $(i=0, \ldots, m)$ 定义在节点向量 $\boldsymbol{T}=\left\{t_{0}, t_{1}, \ldots, t_{m+k}\right\}$ 上. 考虑到 $\boldsymbol{T}$ 有重节点, 将 $\boldsymbol{T}$ 重写作

$$
\boldsymbol{T}=\{\underbrace{t_{0}, \ldots, t_{0}}_{m_{0}}, \ldots, \underbrace{t_{i}, \ldots, t_{i}}_{m_{i}}, \ldots, \underbrace{t_{n}, \ldots, t_{n}}_{m_{n}}\} .
$$

这里 $m_{i}$ 表示节点 $t_{i}$ 的重数并且 $m_{0}=m_{n}=k$.

为了将 C-B-样条曲线从 $k$ 阶升到 $k+1$ 阶, 我们需要找到新的控制顶点 $\left\{\overline{\boldsymbol{P}}_{i}\right\}$ 和节点向量 $\overline{\boldsymbol{T}}$ 使得 $\boldsymbol{P}(t)=\sum_{i=0}^{m} N_{i, k}(t) \cdot \boldsymbol{P}_{i}=\overline{\boldsymbol{P}}(t)=\sum_{i=0}^{\bar{m}} \bar{N}_{i, k+1}(t) \cdot \overline{\boldsymbol{P}}_{i}$. 根据 $\boldsymbol{P}(t)$ 和 $\overline{\boldsymbol{P}}(t)$ 相同的几何与参数性质, 明显有

$$
\overline{\boldsymbol{T}}=\{\underbrace{t_{0}, \ldots, t_{0}}_{m_{0}+1}, \ldots, \underbrace{t_{i}, \ldots, t_{i}}_{m_{i}+1}, \ldots, \underbrace{t_{n}, \ldots, t_{n}}_{m_{n}+1}\} .
$$

对于控制多边形 $\boldsymbol{\Gamma}=\left[\boldsymbol{P}_{0}, \ldots, \boldsymbol{P}_{m}\right]$ 和 $\overline{\boldsymbol{\Gamma}}=\left[\overline{\boldsymbol{P}}_{0}, \ldots, \overline{\boldsymbol{P}}_{m}\right]$, 我们利用升阶算子 $\boldsymbol{A}$ 来升阶曲线, 即满足 $\boldsymbol{A} \cdot \boldsymbol{\Gamma}^{\mathrm{T}}=\overline{\boldsymbol{\Gamma}}^{\mathrm{T}}$. 为了求得升阶算子, 我们需要知道 $\overline{\boldsymbol{P}}_{i}(i=0, \ldots, \bar{m})$ 如何由 $\boldsymbol{P}_{0}, \ldots, \boldsymbol{P}_{m}$ 线性表示.

\section{3 双阶 C-B-样条曲线}

为了求得 $\boldsymbol{A}$, 我们需要引进新的样条. 令

$$
\boldsymbol{T}^{j}=\{\underbrace{t_{0}, \ldots, t_{0}}_{m_{0}+1}, \ldots, \underbrace{t_{j}, \ldots, t_{j}}_{m_{j}+1}, \underbrace{t_{j+1}, \ldots, t_{j+1}}_{m_{j+1}}, \ldots, \underbrace{t_{n}, \ldots, t_{n}}_{m_{n}}\} \quad(j=-1,0, \ldots, n-1) .
$$

下面定义双阶 C-B-样条基函数 $\left\{N_{i, k}^{j}(t)\right\}$, 其在区间 $\left[t_{0}, t_{j+1}\right]$ 上是 $k+1$ 阶, 在区间 $\left[t_{j+1}, t_{n}\right]$ 上是 $k$ 阶. 


\section{1 双阶 $\mathrm{C}-\mathrm{B}$-样条基函数}

令

$$
\boldsymbol{T}^{j}=\{\underbrace{t_{0}, \ldots, t_{0}}_{m_{0}+1}, \ldots, \underbrace{t_{j}, \ldots, t_{j}}_{m_{j}+1}, \underbrace{t_{j+1}, \ldots, t_{j+1}}_{m_{j+1}}, \ldots, \underbrace{t_{n}, \ldots, t_{n}}_{m_{n}}\}
$$

是一个非递减的节点向量, 它通过逐个对 $\boldsymbol{T}$ 的第 $h$ 个节点 $(h=0,1, \ldots, j)$ 插入一个节点来得到.

定义 3 给出定义在空间 $\boldsymbol{T}^{j}=\left\{t_{0}^{j}, t_{1}^{j}, \ldots, t_{s_{j}}^{j}\right\}$ 上的双阶 C-B-样条的初始基函数 $\left(s_{j}=m_{0}+m_{1}+\right.$ $\left.\cdots+m_{n}+j\right)$ :

$$
\begin{aligned}
N_{i, 1}^{j}(t)= \begin{cases}\frac{\sin \left(t-t_{i}^{j}\right)}{\sin \left(t_{i+1}^{j}-t_{i}^{j}\right)}, & \text { 当 } t_{i}^{j} \leqslant t<t_{i+1}^{j} \text { 且 } 0 \leqslant i \leqslant l_{j} ; \\
\frac{\sin \left(t_{i+2}^{j}-t\right)}{\sin \left(t_{i+2}^{j}-t_{i+1}^{j}\right)}, & \text { 当 } t_{i+1}^{j} \leqslant t<t_{i+2}^{j} \text { 且 } 0 \leqslant i \leqslant l_{j}-1 ; \\
1, & \text { 当 } t_{i}^{j} \leqslant t<t_{i+1}^{j} \text { 且 } i>l_{j} ; \\
0, & \text { 其他. }\end{cases} \\
N_{i, 2}^{j}(t)= \begin{cases}\int_{-\infty}^{t}\left[\delta_{i, 1}^{j} N_{i, 1}^{j}(s)-\delta_{i+1,1}^{j} N_{i+1,1}^{j}(s)\right] \mathrm{d} s, & \text { 当 } t_{i}^{j} \leqslant t<t_{i+1}^{j} \text { 且 } 0 \leqslant i \leqslant l_{j}, \\
\frac{\sin \left(t-t_{i}^{j}\right)}{\sin \left(t_{i+1}^{j}-t_{i}^{j}\right)}, & \text { 或 } t_{i+1}^{j} \leqslant t<t_{i+2}^{j} \text { 且 } 0 \leqslant i \leqslant l_{j}-1, \\
\frac{\sin \left(t_{i+2}^{j}-t\right)}{\sin \left(t_{i+2}^{j}-t_{i+1}^{j}\right)}, & \text { 当 } t_{i+2}^{j} \leqslant t<t<t_{i+3}^{j} \text { 且 } 0 \leqslant i \leqslant t_{j}-2 ; \\
0, & \text { 且 } i>l_{j} ;\end{cases}
\end{aligned}
$$

这里 $l_{j}=m_{0}+m_{1}+\cdots+m_{j}+j\left(l_{-1}=-1\right)$.

对 $k \geqslant 3, N_{i, k}^{j}(t)$ 由递归定义:

$$
N_{i, k}^{j}(t)=\int_{-\infty}^{t}\left[\delta_{i, k-1}^{j} N_{i, k-1}^{j}(s)-\delta_{i+1, k-1}^{j} N_{i+1, k-1}^{j}(s)\right] \mathrm{d} s, i=0,1, \ldots, s_{j}-k,
$$

这里

$$
\delta_{i, k}=\left(\int_{-\infty}^{+\infty} N_{i, k}(t) \mathrm{d} t\right)^{-1}
$$

图 1 给出了基函数的一个例子. 这里设 $0 / 0=0$. 如果 $N_{i, k}^{j}(t) \equiv 0$, 定义

$$
\int_{-\infty}^{t} \delta_{i, k}^{j} N_{i, k}^{j}(t) \mathrm{d} t= \begin{cases}1, & \text { 当 } t \geqslant t_{i+k+1}^{j}\left(i \leqslant l_{j}-k\right) \text { 或 } t \geqslant t_{i+k}^{j}\left(i>l_{j}-k\right) ， \\ 0, & \text { 当 } t<t_{i+k+1}^{j}\left(i \leqslant l_{j}-k\right) \text { 或 } t<t_{i+k}^{j}\left(i>l_{j}-k\right) .\end{cases}
$$



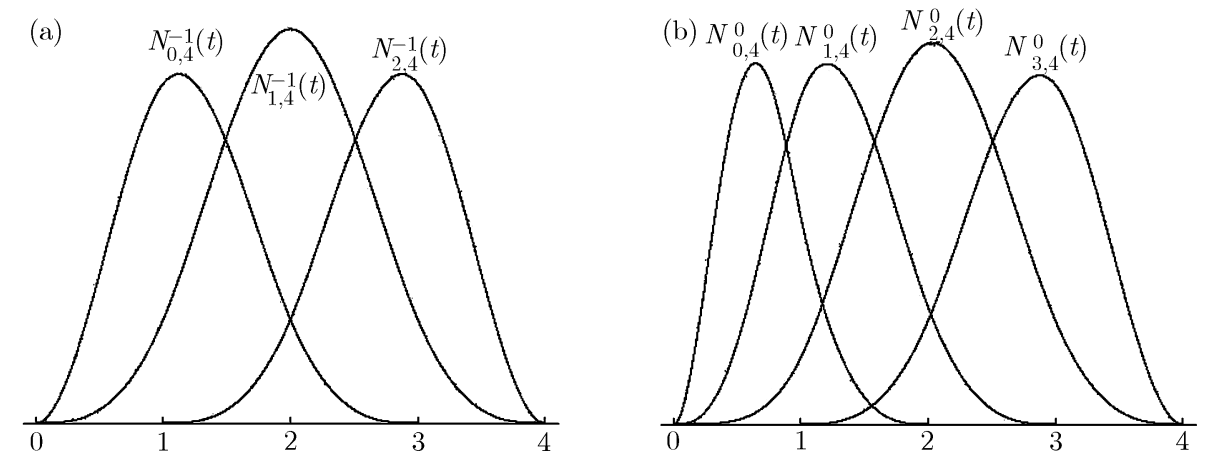

图 1 基函数 $\left\{N_{i, 4}^{-1}(t), N_{i, 4}^{0}(t)\right\}$, 节点向量 $T=\{0,0,1,2,3,4,4\}$

\section{2 双阶 C-B- 样条基的性质}

很明显有 $\boldsymbol{T}^{-1}=\boldsymbol{T}$ 和 $\boldsymbol{T}^{n-1}=\overline{\boldsymbol{T}}$.

定理 1 假定 $\left\{N_{i, k}(t)\right\}$ 和 $\left\{\bar{N}_{i, k+1}(t)\right\}$ 分别是定义在 $\boldsymbol{T}$ 和 $\overline{\boldsymbol{T}}$ 上的 C-B-样条基函数. $\left\{N_{i, k}^{-1}(t)\right\}$ 和 $\left\{N_{i, k}^{n-1}(t)\right\}$ 分别是定义在 $\boldsymbol{T}^{-1}$ 和 $\boldsymbol{T}^{n-1}$ 上的双阶 C-B-样条基函数. 那么我们有 $N_{i, k}^{-1}(t)=N_{i, k}(t)$, $N_{i, k}^{n-1}(t)=\bar{N}_{i, k+1}(t)$.

证明 根据数学归纳法: 当 $k=1$ 时, 定理明显成立.

假定当 $k>1$, 有 $N_{i, k}^{-1}(t)=N_{i, k}(t)$. 据假定, 则有 $\delta_{i, k}^{-1}=\delta_{i, k}, N_{i, k}^{-1}(t)=N_{i, k}(t)$. 那么就有 $N_{i, k+1}^{-1}(t)=$ $N_{i, k+1}(t)$. 接着容易推出在 $\overline{\boldsymbol{T}}$ 上 $\bar{N}_{i, 2}(t)=N_{i, 1}^{n-1}(t)$. 最后由上述的方法, 能推出 $N_{i, k}^{n-1}(t)=\bar{N}_{i, k+1}(t)$.

由定理 1 , 如果知道 $N_{i, k}^{-1}(t)$ 和 $N_{i, k}^{n-1}(t)$ 的关系, 就能推导出 $N_{i, k}(t)$ 和 $\bar{N}_{i, k+1}(t)$ 之间的变换公式 以及升阶算子 $\boldsymbol{A}$. 进一步, 有下面的定理.

定理 $2\left\{N_{i, k}^{j-1}(t)\right\}$ 和 $\left\{N_{i, k}^{j}(t)\right\}$ 的定义由 $(1) \sim(5)$ 式给出, 并且 $\boldsymbol{T}^{j}=\boldsymbol{T}^{j-1}+\left\{t_{j}\right\}$. 它们相互之 间的变换公式如下:

$$
N_{i, k}^{j-1}(t)=\left(1-a_{i, k}^{j}\right) N_{i, k}^{j}(t)+a_{i+1, k}^{j} N_{i+1, k}^{j}(t),
$$

这里 $\delta_{i, k}^{j}$ 由 (4) 式定义, 而 $a_{i, k}^{j}$ 定义如下:

$$
a_{i, 1}^{j}=\left\{\begin{array}{ll}
0, & i<l_{j}, \\
1, & i \geqslant l_{j} ;
\end{array} \quad a_{i, l+1}^{j}= \begin{cases}0, & i<l_{j}-l, \\
\frac{a_{i+1, l}^{j} \delta_{i, l}^{j-1}}{\delta_{i+1, l}^{j}}, & l_{j}-l \leqslant i \leqslant l_{j}-1(l \geqslant 1), \\
1, & i \geqslant l_{j} .\end{cases}\right.
$$

证明 当 $k=1$ 时, 明显成立. 假定定理对 $k>1$ 也成立, 那么有 $\int_{-\infty}^{+\infty} N_{i, k}^{j-1}(t) \mathrm{d} t=\int_{-\infty}^{+\infty}\left[\left(1-a_{i, k}^{j}\right)\right.$ $\left.N_{i, k}^{j}(t)+a_{i+1, k}^{j} N_{i+1, k}^{j}(t)\right] \mathrm{d} t$. 那么有

$$
\delta_{i, k}^{j-1}=\frac{\delta_{i, k}^{j} \delta_{i+1, k}^{j}}{\left(1-a_{i, k}^{j}\right) \delta_{i+1, k}^{j}+a_{i+1, k}^{j} \delta_{i, k}^{j}} .
$$

对 $k+1$,

$$
N_{i, k+1}^{j-1}(t)=\int_{-\infty}^{t}\left[\delta_{i, k}^{j-1} N_{i, k}^{j-1}(s)-\delta_{i+1, k}^{j-1} N_{i+1, k}^{j-1}(s)\right] \mathrm{d} s
$$




$$
\begin{aligned}
= & \int_{-\infty}^{t}\left\{\frac{\delta_{i, k}^{j} \delta_{i+1, k}^{j}\left[\left(1-a_{i, k}^{j}\right) N_{i, k}^{j}(s)+a_{i+1, k}^{j} N_{i+1, k}^{j}(s)\right]}{\left(1-a_{i, k}^{j}\right) \delta_{i+1, k}^{j}+a_{i+1, k}^{j} \delta_{i, k}^{j}}-\frac{\delta_{i+1, k}^{j} \delta_{i+2, k}^{j}\left(1-a_{i+1, k}^{j}\right) N_{i+1, k}^{j}(s)}{\left(1-a_{i+1, k}^{j}\right) \delta_{i+2, k}^{j}+a_{i+2, k}^{j} \delta_{i+1, k}^{j}}\right. \\
& \left.-\frac{\delta_{i+1, k}^{j} \delta_{i+2, k}^{j} a_{i+2, k}^{j} N_{i+2, k}^{j}(s)}{\left(1-a_{i+1, k}^{j}\right) \delta_{i+2, k}^{j}+a_{i+2, k}^{j} \delta_{i+1, k}^{j}}\right\} \mathrm{d} s \\
= & {\left[1-\frac{a_{i+1, k}^{j} \delta_{i, k}^{j}}{\left(1-a_{i, k}^{j}\right) \delta_{i+1, k}^{j}+a_{i+1, k}^{j} \delta_{i, k}^{j}}\right] N_{i, k+1}^{j}(t)+\frac{a_{i+2, k}^{j} \delta_{i+1, k}^{j}}{\left(1-a_{i+1, k}^{j}\right) \delta_{i+2, k}^{j}+a_{i+2, k}^{j} \delta_{i+1, k}^{j}} N_{i+1, k+1}^{j}(t) } \\
= & \left(1-a_{i, k+1}^{j}\right) N_{i, k+1}^{j}(t)+a_{i+1, k+1}^{j} N_{i+1, k+1}^{j}(t) .
\end{aligned}
$$

这就是说定理对 $k+1$ 也成立. 证毕.

图 2 和 3 表明了不同节点下双阶样条基函数的线性变化关系.

推论 $1 \quad 0 \leqslant a_{i, k}^{j} \leqslant 1$.

现在我们可以知道双阶 C-B-样条和一般 C-B-样条具有相同的性质.

(1) 归一性: $\sum_{i} N_{i, k}^{j}(t)=1$.

(2) 正性: 对 $t \in\left(t_{i}^{j}, t_{i+k}^{j}\right)\left(i>l_{j}-k\right)$ 或 $t \in\left(t_{i}^{j}, t_{i+k+1}^{j}\right)\left(i \leqslant l_{j}-k\right)$, 有 $N_{i, k}^{j}(t)>0$.

(3) 全局线性独立性: $\left\{N_{i, k}^{j}(t)\right\}$ 在 $\boldsymbol{T}^{j}\left(N_{i, k}^{j}(t) \neq 0\right)$ 上是线性独立的.

\section{3 双阶 C-B-样条曲线}

基于双阶 C-B-样条基函数, 构造它相应的曲线.

定义 4 一条定义在 $\boldsymbol{T}^{j}$ 上的 $k$ 阶双阶 C-B-样条曲线 $\boldsymbol{P}^{j}(t)=\sum_{i=0}^{s_{j}-k-1} N_{i, k}^{j}(t) \cdot \boldsymbol{P}_{i}^{j}\left(t_{0} \leqslant t \leqslant t_{n}\right)$, 这 里 $\left\{\boldsymbol{P}_{i}^{j}\right\}$ 是控制顶点和 $\left\{N_{i, k}^{j}(t)\right\}$ 是双阶 C-B-样条基函数.

容易看出, 曲线 $\boldsymbol{P}^{-1}(t)$ 就是定义在节点 $\boldsymbol{T}^{-1}=\boldsymbol{T}$ 上的 $k$ 阶 C-B- 样条曲线, 而曲线 $\boldsymbol{P}^{n-1}(t)$ 是 定义在节点 $\boldsymbol{T}^{n-1}=\overline{\boldsymbol{T}}$ 上的 $k+1$ 阶 C-B- 样条曲线.

\section{C-B-样条曲线的升阶算子}

根据第 3 节的双阶 C-B-样条, 能计算出升阶算子 $\boldsymbol{A}$.

\section{1 升阶算子}

注意到双阶 C-B-样条基函数 $\left\{N_{i, k}^{j-1}(t)\right\}$ 能用 $\left\{N_{i, k}^{j}(t)\right\}$ 线性表示, 得到 $\left\{\boldsymbol{P}_{i}^{j-1}\right\}$ 和 $\left\{\boldsymbol{P}_{i}^{j}\right\}$ 之间的变 换公式.

定理 $3 \boldsymbol{P}^{j-1}(t)$ 是定义在 $\boldsymbol{T}^{j-1}$ 上的一条双阶 C-B-样条曲线. 曲线 $\boldsymbol{P}^{j}(t)$ 是通过对 $\boldsymbol{T}^{j-1}$ 嵌入 节点 $\left\{t_{j}\right\}$ 后得到的双阶样条曲线. 显然它们是相同的曲线, 那么其控制顶点 $\left\{\boldsymbol{P}_{i}^{j-1}\right\},\left\{\boldsymbol{P}_{i}^{j}\right\}$ 满足

$$
\boldsymbol{P}_{i}^{j}=\left(1-a_{i, k}^{j}\right) \boldsymbol{P}_{i}^{j-1}+a_{i, k}^{j} \boldsymbol{P}_{i-1}^{j-1},
$$

这里 $a_{i, k}^{j}$ 由 (7) 式定义.

根据定理 3, 将上式重写作 $\boldsymbol{P}_{i}^{j}=\boldsymbol{X} \cdot\left[\boldsymbol{P}_{0}^{j-1}, \boldsymbol{P}_{1}^{j-1}, \ldots, \boldsymbol{P}_{m+j}^{j-1}\right]^{\mathrm{T}}$, 这里 $\boldsymbol{X}=\left[0, \ldots, 0, a_{i, k}^{j}, 1-a_{i, k}^{j}, 0\right.$, $\ldots, 0]$. 

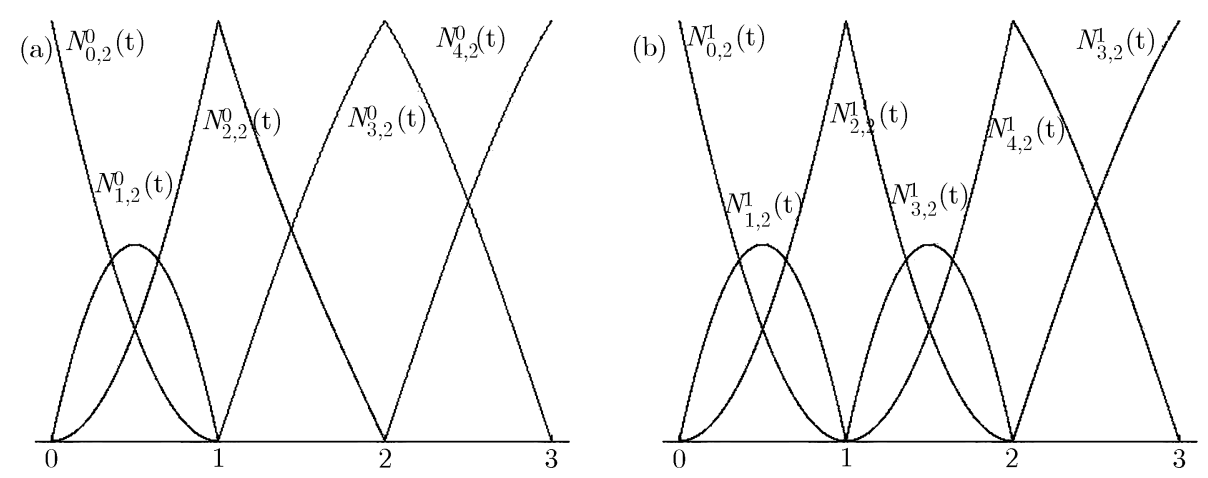

图 2 基函数 $\left\{N_{i, 2}^{0}(t)\right\}$ 和 $\left\{N_{i, 2}^{1}(t)\right\}$, 节点向量 $T=\{0,0,1,2,3,3\}$
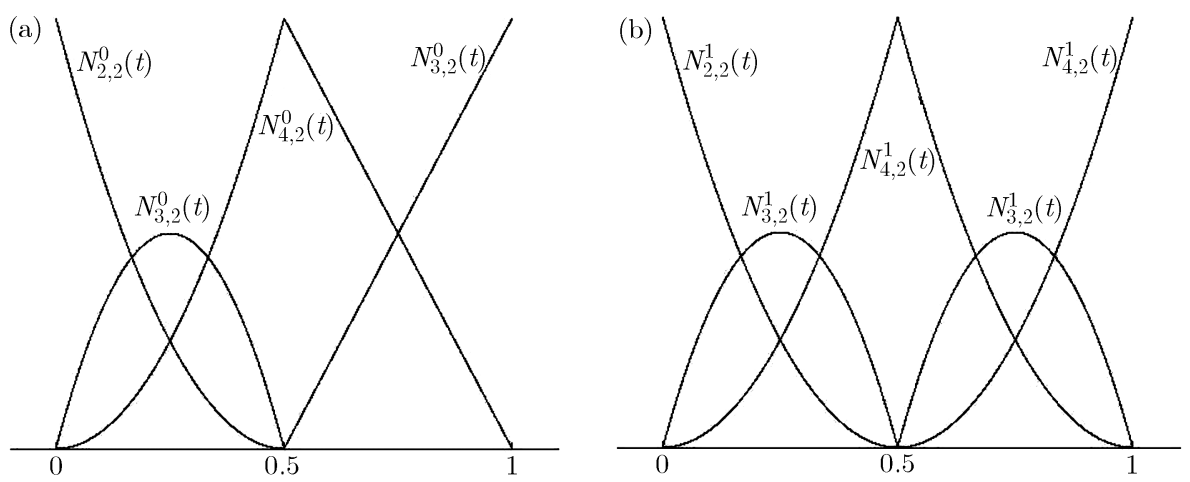

图 3 基函数 $\left\{N_{i, 2}^{0}(t)\right\}$ 和 $\left\{N_{i, 2}^{1}(t)\right\}$, 节点向量 $T=\{0,0,0,0,0.5,1,1,1,1\}$

对于 $\boldsymbol{\Gamma}^{j}=\left[\boldsymbol{P}_{0}^{j}, \boldsymbol{P}_{1}^{j}, \ldots, \boldsymbol{P}_{m+j+1}^{j}\right]$ 和 $\boldsymbol{\Gamma}^{j-1}=\left[\boldsymbol{P}_{0}^{j-1}, \boldsymbol{P}_{1}^{j-1}, \ldots, \boldsymbol{P}_{m+j}^{j-1}\right]$, 记 $\left(\boldsymbol{\Gamma}^{j}\right)^{\mathrm{T}}=\boldsymbol{X}_{j} \cdot\left(\boldsymbol{\Gamma}^{j-1}\right)^{\mathrm{T}}$, 这里

$$
\boldsymbol{X}_{j}=\left(\begin{array}{ccccc}
1-a_{0, k}^{j} & 0 & \cdots & \cdots & 0 \\
a_{1, k}^{j} & 1-a_{1, k}^{j} & \cdots & \cdots & 0 \\
\cdots & \cdots & \cdots & \cdots & \cdots \\
0 & 0 & \cdots & a_{m+j, k}^{j} & 1-a_{m+j, k}^{j} \\
0 & 0 & \cdots & \cdots & a_{m+j+1, k}^{j}
\end{array}\right), \quad\left(\begin{array}{c}
(m+j+2) \times(m+j+1) \\
j=0,1, \ldots, n-1
\end{array}\right) .
$$

现在我们可以利用矩阵 $\boldsymbol{X}_{j}$ 将 $\boldsymbol{P}^{j-1}(t)$ 的控制顶点更新到 $\boldsymbol{P}^{j}(t)$ 的控制顶点. 因为 $\boldsymbol{P}^{0}(t)=\boldsymbol{P}(t)$, $\boldsymbol{P}^{n-1}(t)=\overline{\boldsymbol{P}}(t)$, 得出 $\boldsymbol{\Gamma}^{\mathrm{T}}=\left(\boldsymbol{\Gamma}^{n-1}\right)^{\mathrm{T}}=\boldsymbol{X}_{n-1} \cdot \boldsymbol{X}_{n-2} \cdots \boldsymbol{X}_{0} \cdot \boldsymbol{\Gamma}^{\mathrm{T}}=\boldsymbol{A} \cdot \boldsymbol{\Gamma}^{\mathrm{T}}$, 这里 $\boldsymbol{A}=\boldsymbol{X}_{n-1} \boldsymbol{X}_{n-2} \cdots \boldsymbol{X}_{0}$.

$\boldsymbol{A}$ 就是要求的升阶算子, 因此我们能通过计算 $\left\{\boldsymbol{X}_{j}\right\}_{j=0}^{n-1}$ 来得到 $\boldsymbol{A}$.

\section{2 举例}

图 4 是一个升阶的例子.

\section{3 复杂性分析}

表 1 将升阶算子与其他算法 ${ }^{[17]}$ 进行比较. 


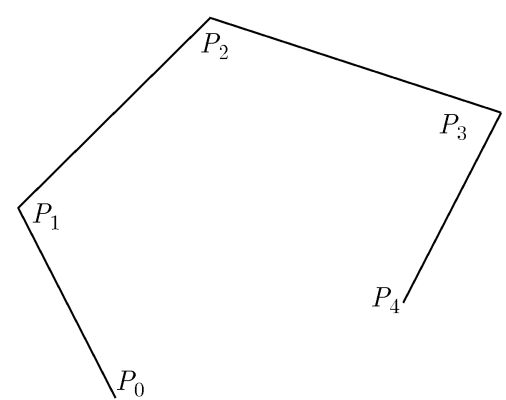

(a)

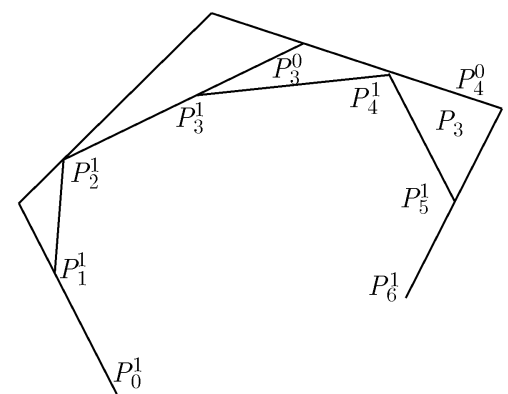

(c)

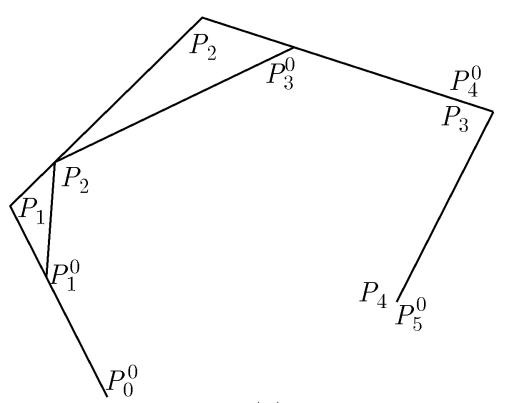

(b)

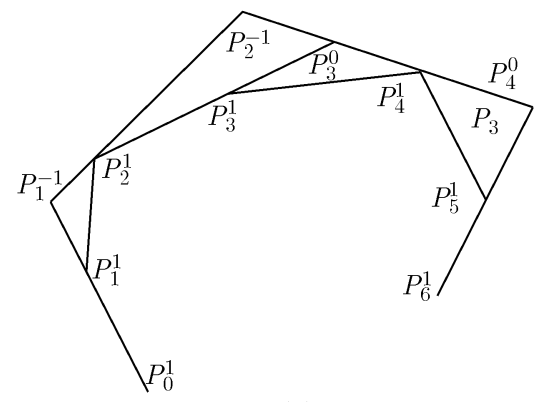

(d)

图 4 升阶的例子. 定义在 $T=\{0,0,0,0,0.6,1,1,1,1\}$ 上的 5 阶 C-B-样条

表 1 升阶一次

\begin{tabular}{cc}
\hline \hline 操作 & Peautzsch 算法 \\
\hline+- & $(m-k+1)(10 k-8)+k$ \\
$\times$ & $(m-k+1)(5 k-2)+2 k$ \\
\hline 快速升阶算法 & 本文算法 \\
\hline $6(m-k+1)(k-1)+2 k(k-1)$ & $(m-k+1)(k+1)$ \\
$(m-k+1)(2 k-1)+k(k-1) / 2$ & $2(m-k+1)(k+1)$ \\
\hline \hline
\end{tabular}

从表 1 看出, 我们的运算比其他算法更有效率.

\section{5 收敛性定理}

根据上面的定理, 将 C-B-样条曲线 $\boldsymbol{P}(t)$ 从 $k$ 阶升阶至 $k+1$ 阶是一个对控制多边形割角的过程. 当升阶的阶数足够高时, 新的控制多边形序列将收玫到初始的样条曲线. 这个性质对 Bézier 曲线显然 是成立的. 我们将表明 C-B-样条曲线也有相同的性质.

为方便, 引进一些符号:

(1) $N_{i, k}(t)$ 是定义在 $\boldsymbol{T}$ 上的 $k$ 阶 C-B-样条基函数; $N_{i, k}^{(j)}(t)$ 表示 $N_{i, k}(t)$ 的 $j$ 次导数.

(2) $\hat{N}_{i, K}(t)$ 是升阶后 $K$ 阶 C-B-样条基函数; $\hat{\boldsymbol{T}}$ 是升阶后的节点向量.

$$
\hat{\boldsymbol{T}}=\{\underbrace{\tau_{0}, \ldots, \tau_{0}}_{r_{0}}, \ldots, \underbrace{\tau_{i}, \ldots, \tau_{i}}_{r_{i}}, \ldots, \underbrace{\tau_{n}, \ldots, \tau_{n}}_{r_{n}}\}
$$




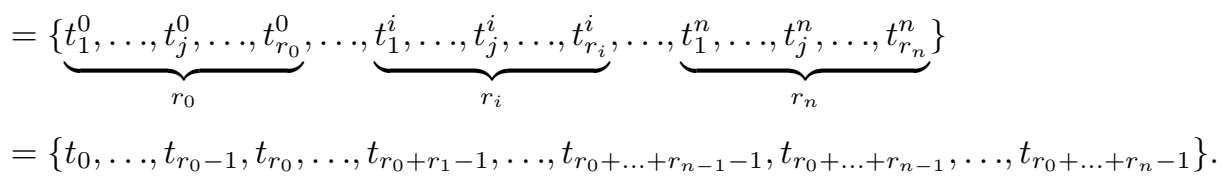

这样可以推出 $t_{j}^{i}=t_{r_{0}+r_{1}+\cdots+r_{i-1}+j-1}$. 假定 $L(i, j)=r_{0}+r_{1}+\cdots+r_{i-1}+j-1$, 那么有 $t_{j}^{i}=t_{L(i, j)}$.

(3) $u_{K}$ 表示 $\hat{N}_{i, K}(t)$ 的非零节点区间个数.

在接下来的证明中, 将用到 C-Bézier ${ }^{[23,24]}$ 基函数的定义与性质.

\section{1 积分估计}

下面两个引理是明显成立的.

引理 1 对 $k$ 阶 C-B- 样条基函数 $N_{i, k}(t)$, 有 $N_{i, k}^{(j)}(t)=\sum_{l=0}^{j} \alpha_{j, l} \cdot N_{i+l, k-j}(t)$, 这里 $\alpha_{j, l}$ 定义为 $\alpha_{0,0}=1, \alpha_{j, 0}=\delta_{i, k-j} \alpha_{j-1,0}, \alpha_{j, j}=-\delta_{i+j, k-j} \alpha_{j-1, j-1}, \alpha_{j, l}=\delta_{i+l, k-j}\left(\alpha_{j-1, l}-\alpha_{j-1, l-1}\right)$.

引理 2 对 $k$ 阶 C-B-样条基 $N_{i, k}(t)$, 满足: 当 $t=t_{i}\left(j=0,1, \ldots, k-1-m_{i}\right)$ 时, 有 $N_{i, k}^{(j)}\left(t_{i}\right)=0$, $N_{i, k}^{k-m_{i}}\left(t_{i}\right)>0$; 当 $t=t_{i+k}\left(j=0,1, \ldots, k-1-n_{i+k}\right)$ 时, $N_{i, k}^{(j)}\left(t_{i+k}\right)=0, N_{i, k}^{k-n_{i+k}}\left(t_{i+k}\right)<0$. 这里 $m_{i}, n_{i}$ 分别 是是节点的向前和向后重数 (满足 $t_{i}=\ldots=t_{i+m_{i}-1}<t_{i+m_{i}}, t_{i-n_{i}}<t_{i-n_{i}+1}=\cdots=t_{i}$ ).

引理 3 一条定义在 $\boldsymbol{T}$ 上的 $k$ 阶 C-B-样条曲线为 $\boldsymbol{P}(t)=\sum_{i=0}^{m} N_{i, k}(t) \cdot \boldsymbol{P}_{i}$. 将曲线 $\boldsymbol{P}(t)$ 从 $k$ 阶 升阶至 $K(K>2 k)$ 时, 定义在新的节点向量 $\hat{\boldsymbol{T}}$ 上的 $K$ 阶 C-B-样条基函数 $\hat{N}_{i, K}(t)$, 其非零局部支撑 区间的个数不超过 2 . 并且只包含有 2 个非零局部支撑区间的基函数的个数不超过 $n k$, 这里 $n$ 是 $\hat{\boldsymbol{T}}$ 中的非零节点区间个数.

证明 对 $\hat{N}_{i, K}(t)$, 其支撑区间是 $\left(t_{i}, t_{i+K}\right)$. 假定 $t_{i}=t_{j}^{l}, t_{i}$ 是对应到 $\hat{\boldsymbol{T}}$ 中 $\tau_{l}$ 的第 $j$ 个节点, 这样 只需证明 $i+K<L(l+3,1)$. 因为 $i+K=L(l, j)+K=\left(r_{0}+\cdots+r_{l-1}+j-1\right)+K, j-1<r_{l}$ 和 $K<r_{l+1}+r_{l+2}$, 则有 $i+K<L(l+3,1)$. 即 $u_{K} \leqslant 2$. 当 $j<r_{l}-k$, 因为 $r_{l+1}>K-k, i+K<r_{0}+\cdots+r_{l+1}=L(l+2,1)$, 故有 $t_{i+K}<t_{1}^{l+2}$. 即当 $j<r_{l}-k$, 有 $u_{K}=1$. 由此断定只含有 2 个非零局部支撑区间的基函数个数不超过 $n k$. 证毕.

对 C-B-样条基 $\hat{N}_{i, K}(t)$, 当 $t_{i}=t_{j}^{l}$, 根据引理 3 , 它的非零支撑区间是 $\left(\tau_{l}, \tau_{l+1}\right)$ 或者 $\left(\tau_{l}, \tau_{l+2}\right)$. 如果 $u_{K}=1, \hat{N}_{i, K}(t)$ 就是定义在 $\left(\tau_{l}, \tau_{l+1}\right)^{[3]}$ 上的 C-Bézier 基函数; 如果 $u_{K}=2$, 根据 C-B- 样条的嵌入节点 定理 ${ }^{[3]}$ (即插入一个节点之后, 有 $\hat{N}_{i, K}(t)=\zeta_{i, K} \hat{N}_{i, K}^{1}(t)+\eta_{i+1, K} \hat{N}_{i+1, K}^{1}(t)$, 这里 $\hat{N}_{i, K}^{1}(t)$ 是插入节点后的 C-B-样条基函数), 分别在 $\tau_{l}, \tau_{l+1}, \tau_{l+2}$ 插入节点直到 $K$ 重之后, 有 $\hat{N}_{i, K}(t)=\sum_{j=M(i)}^{j=M(i)+C} \alpha_{j, K} \hat{N}_{j, K}^{q}(t)$, 这里 $q$ 是插入频率, $\hat{N}_{j, K}^{q}(t)$ 是插入节点后的新的 C-B-样条基函数, 以及 $\alpha_{j, K} \geqslant 0$. 也就是说 $\hat{N}_{i, K}(t)$ 能 够用有限个新的 C-B-样条基函数非负线性表示. 因为 $\tau_{l}, \tau_{l+1}, \tau_{l+2}$ 的重数都是 $K$, 根据 C-Bézier 基的 定义, $\hat{N}_{j, K}^{q}(t)$ 就是定义在 $\left[\tau_{l}, \tau_{l+1}\right]$ 或 $\left[\tau_{l+1}, \tau_{l+2}\right]$ 上的 C-Bézier 基函数. 所以, C-B-样条基函数 $\hat{N}_{i, K}(t)$ 可以用有限个 C-Bézier 基函数非负线性表出.

定理 4 设将 $k$ 阶 C-B-样条曲线升阶到 $K(K>2 k)$ 阶之后的节点向量为 $\hat{\boldsymbol{T}}$, 则定义在 $\hat{\boldsymbol{T}}$ 上的 $K$ 阶 C-B-样条基函数一定能够用不超过 $2 k$ 个 $K$ 阶 C-Bézier 基函数线性表出.

证明 因为 $\left(t_{i}, t_{i+K}\right)$ 是 $\hat{N}_{i, K}(t)$ 的支撑区间, 在 $\hat{\boldsymbol{T}}$ 中 $t_{i}=t_{j}^{l}$, 也就有 $i=L(l, j)=\left(r_{0}+\cdots+r_{l-1}+j-1\right)$, $i+K=\left(r_{0}+\cdots+r_{l-1}+j-1\right)+K$. 令 $B_{i, K-1}^{l}(t)(i=0, \ldots, K-1)$ 与 $B_{j, K-1}^{l+1}(t)(j=0, \ldots, K-1)$ 分别为区间 $\left[\tau_{l}, \tau_{l+1}\right]$ 和 $\left[\tau_{l+1}, \tau_{l+2}\right]$ 上的 C-Bézier 基函数. 据前面分析, 有

$$
\hat{N}_{i, K}(t)=\sum_{m=0}^{K-1} \alpha_{i, m} B_{m, K-1}^{l}(t)+\sum_{h=0}^{K-1} \beta_{i, h} B_{h, K-1}^{l+1}(t) .
$$


如果 $u_{K}=1$, 取 $t \in\left[\tau_{l+1}, \tau_{l+2}\right], \hat{N}_{i, K}(t)=0$. 考虑到线性独立性, $\beta_{i, h}=0(h=0, \ldots, K-1) . t_{i}$ 在 $\hat{\boldsymbol{T}}$ 中的向后 重数为 $\left(r_{0}+\cdots+r_{l}\right)-i=r_{l}-j+1$, 而 $t_{i+K}$ 在 $\hat{\boldsymbol{T}}$ 中向前重数为 $(i+K)-\left(r_{0}+\cdots+r_{l}\right)+1=j+K-r_{l}$. 现在对 (9) 式两边进行求导, 根据引理 2 和 C-Bézier 基函数在端点处的性质 [23] 有 $\alpha_{i, m}=0\left(m \leqslant(K-1)-\left(r_{l}-\right.\right.$ $j+1))$. 而且 $\alpha_{i, m}=0\left(m \geqslant j+K-r_{l}\right)$. 故有 $\hat{N}_{i, K}(t)=B_{K+j-r_{l}-1, K-1}^{l}(t)$. 所以 $\hat{N}_{i, K}(t)$ 实际上就是定义在 $\left[\tau_{l}, \tau_{l+1}\right]$ 上的 C-Bézier 基函数. 如果 $u_{K}=2$, 根据引理 3 有 $r_{l}-k \leqslant j \leqslant r_{l}, t_{i}$ 在 $\hat{\boldsymbol{T}}$ 的向后重数为 $r_{l}-j+1$ 以及 $t_{i+K}$ 的向前重数为 $j+K-r_{l}-r_{l+1}$. 由引理 2 和上面的方法, 推得 $\alpha_{i, m}=0\left(m \leqslant(K-1)-\left(r_{l}-j+1\right)\right)$ 和 $\beta_{i, h}=0\left(h \geqslant K-1-\left[(K-1)-\left(j+K-r_{l}-r_{l+1}\right)\right]\right)$. 那么有 $\hat{N}_{i, K}(t)=\sum_{m=(K-1)-\left(r_{l}-j\right)}^{K-1} \alpha_{i, m} B_{m, K-1}^{l}(t)+$ $\sum_{h=0}^{j+K-r_{l}-r_{l+1}-1} \beta_{i, h} B_{h, K-1}^{l+1}(t)$. 根据前面的分析, $r_{l}-j \leqslant k, j+K-r_{l}-r_{l+1}-1 \leqslant K-r_{l+1}-1 \leqslant k-1$, 以及 $0 \leqslant \alpha_{i, m} \leqslant 1,0 \leqslant \beta_{i, h} \leqslant 1$. 所以 $\hat{N}_{i, K}(t)$ 能够用不超过 $2 k$ 个 $K$ 阶 C-Bézier 基函数非负线性表出. 证毕.

定理 5 对 $k$ 阶 C-B-样条曲线进行升阶. 当升高的阶数足够高 $(K>2 k)$, C-B-样条基函数 $\hat{N}_{i, K}(t)$ 的积分有以下估计: $\int_{-\infty}^{+\infty} \hat{N}_{i, K}(t) \mathrm{d} t \leqslant \frac{5 U_{1}}{K}$, 这里 $U_{1}=2 k \cdot \max \left\{\tau_{j+1}-\tau_{j} \mid j=0,1, \ldots, n-1\right\}$ 是最大节点区间 长度的 $2 k$ 倍.

证明 对定义于区间 $[0, \alpha]$ 上的 C-Bézier 基函数 $B_{i, K}(t)$, 有积分估计 ${ }^{[24]}: \int_{0}^{\alpha} B_{i, K}(t) \mathrm{d} t \leqslant \frac{5 \alpha}{K+1}$. 根据定理 4 , 有

$$
\begin{aligned}
\int_{-\infty}^{+\infty} \hat{N}_{i, K}(t) \mathrm{d} t & =\int_{-\infty}^{+\infty}\left[\sum_{m=(K-1)-\left(r_{l}-j\right)}^{K-1} \alpha_{i, m} B_{m, K-1}^{l}(t)+\sum_{h=0}^{j+K-r_{l}-r_{l+1}-1} \beta_{i, h} B_{h, K-1}^{l+1}(t)\right] \mathrm{d} t \\
& \leqslant \frac{2 k \cdot 5 \cdot \max \left\{\tau_{j+1}-\tau_{j} \mid j=0, \ldots, n-1\right\}}{K}=\frac{5 U_{1}}{K} .
\end{aligned}
$$

证毕.

\section{2 控制多边形的收敛性}

定理 6 一条 $k$ 阶 C-B-样条曲线为 $\boldsymbol{P}(t)=\sum_{i=0}^{m} N_{i, k}(t) \cdot \boldsymbol{P}_{i}$. 将它升阶到 $K(K>2 k)$ 阶, 曲线变 成 $\boldsymbol{P}(t)=\sum_{i=0}^{M} \hat{N}_{i, K}(t) \cdot \boldsymbol{P}_{i}^{K}$, 那么存在 $U>0$ 满足 $\left\|\boldsymbol{P}_{i+1}^{K}-\boldsymbol{P}_{i}^{K}\right\| \leqslant \frac{U}{K-1}(i=0, \ldots, M-1)$.

证明 C-B-样条曲线为 $\boldsymbol{P}(t)=\sum_{i=0}^{m} N_{i, k}(t) \cdot \boldsymbol{P}_{i}^{k}=\sum_{i=0}^{M} \hat{N}_{i, K}(t) \cdot \boldsymbol{P}_{i}^{K}$, 这里 $\boldsymbol{P}_{i}^{k}=\boldsymbol{P}_{i}$. 对前面的公 式两边求导, $\boldsymbol{P}^{\prime}(t)=\sum_{i=0}^{m-1} \delta_{i+1, k-1} N_{i+1, k-1}(t) \cdot\left(\boldsymbol{P}_{i+1}^{k}-\boldsymbol{P}_{i}^{k}\right)=\sum_{i=0}^{M-1} \hat{N}_{i+1, K-1}(t) \cdot\left(\boldsymbol{P}_{i+1}^{K}-\boldsymbol{P}_{i}^{K}\right)$. 这个式 子可以看作是低一阶的 C-B-样条曲线. 右边的曲线可以通过将左边曲线升阶 $K-k$ 次得到. 所以 右边的控制顶点 $\left\{\delta_{i+1, K-1}\left(\boldsymbol{P}_{i+1}^{K}-\boldsymbol{P}_{i}^{K}\right)\right\}$ 能够通过对左边控制顶点 $\left\{\delta_{i+1, k-1}\left(\boldsymbol{P}_{i+1}^{k}-\boldsymbol{P}_{i}^{k}\right)\right\}$ 进行割角得 到. 也就有 $\left\|\delta_{i+1, K-1}\left(\boldsymbol{P}_{i+1}^{K}-\boldsymbol{P}_{i}^{K}\right)\right\| \leqslant U_{2}, U_{2}=\max _{0 \leqslant i \leqslant m-1}\left\{\left\|\delta_{i+1, k-1}\left(\boldsymbol{P}_{i+1}^{k}-\boldsymbol{P}_{i}^{k}\right)\right\|\right\}$. 根据定理 5 , 有 $\left\|\boldsymbol{P}_{i+1}^{K}-\boldsymbol{P}_{i}^{K}\right\| \leqslant \delta_{i+1, K-1}^{-1} U_{1} \leqslant U /(K-1)$, 这里 $U=5 U_{1} U_{2}$.

定理 7 对 C-B-样条曲线 $\boldsymbol{P}(t)=\sum_{i=0}^{m} N_{i, k}(t) \cdot \boldsymbol{P}_{i}$ 进行升阶, 相当于将升阶算子 $\boldsymbol{A}$ 作用于控制 多边形 $\boldsymbol{\Gamma}$ 上. 最后, 当 $r \rightarrow \infty$ 时, 其控制多边形序列 $\boldsymbol{A}^{0} \boldsymbol{\Gamma}, \boldsymbol{A}^{1} \boldsymbol{\Gamma}, \ldots, \boldsymbol{A}^{r} \boldsymbol{\Gamma}$ 将收敛到初始的 C-B-样条 曲线.

证明 将曲线的阶数从 $k$ 阶升阶至 $r(r>2 k)$ 阶, 升阶后的曲线为 $\boldsymbol{P}(t)=\sum_{i=0}^{M} \hat{N}_{i, r}(t) \cdot \boldsymbol{P}_{i}^{r}$, 这里 $\hat{N}_{i, r}(t)$ 是定义在

$$
\hat{\boldsymbol{T}}=\{\underbrace{\tau_{0}, \ldots, \tau_{0}}_{r_{0}}, \ldots, \underbrace{\tau_{i}, \ldots, \tau_{i}}_{r_{i}}, \ldots, \underbrace{\tau_{n}, \ldots, \tau_{n}}_{r_{n}}\}
$$

上的. 由定理 6 , 当升高的阶数足够大时, 相邻控制顶点的距离就会足够小. 即对 $\forall \varepsilon>0, \exists N_{1}>0$, 当 $r>N_{1}$, 有

$$
\left\|\boldsymbol{P}_{i+1}^{r}-\boldsymbol{P}_{i}^{r}\right\| \leqslant \frac{\varepsilon}{2^{(n+1) k+1}} \quad(i=0,1, \ldots, M-1) .
$$




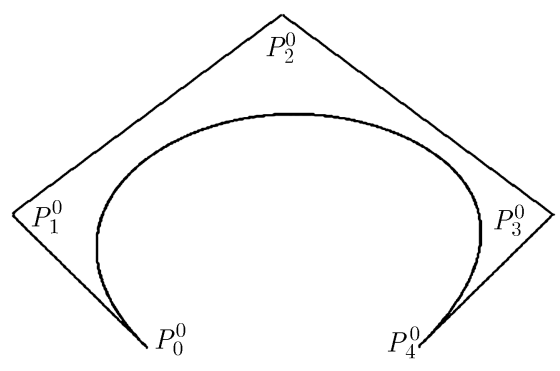

(a) 初始C-B-样条曲线和控制多边形

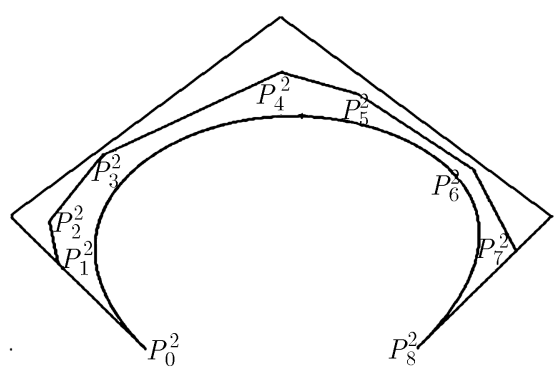

(c) 升阶两次以后的控制多边形

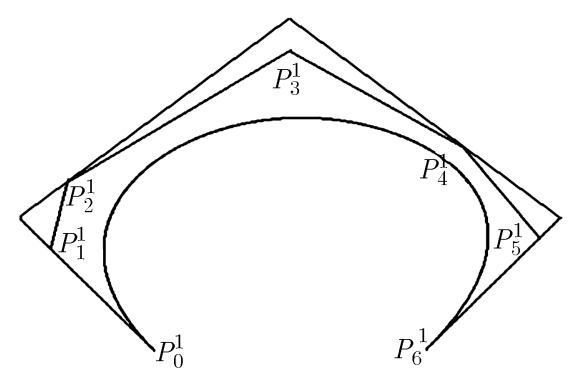

(b) 升阶一次以后的控制多边形

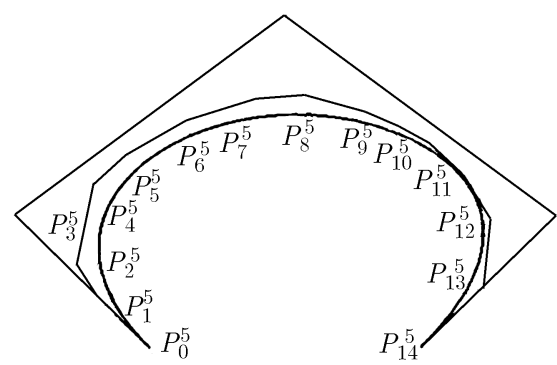

(d) 升阶五次以后的控制多边形

图 5 C-B-样条曲线的几何构造过程

现在如果将一个节点 $\tau_{i}$ 插入 $\boldsymbol{P}(t)$, 新的曲线为 $\boldsymbol{Q}(t)=\sum_{i=0}^{M+1} \hat{N}_{i, r}(t) \cdot \boldsymbol{Q}_{i, r} . \boldsymbol{\Gamma}_{1}=\boldsymbol{P}_{0}^{r} \cdots \boldsymbol{P}_{M}^{r}$ 和 $\boldsymbol{\Gamma}_{2}=$ $\boldsymbol{Q}_{0}^{r} \cdots \boldsymbol{Q}_{M+1}^{r}$ 分别为插入前后的控制多边形. 根据插入节点定理 ${ }^{[3]}$, 控制顶点 $\left\{\boldsymbol{Q}_{i}^{r}\right\}_{i=0}^{M+1}$ 能够通过对控 制多边形 $\boldsymbol{\Gamma}_{1}$ 割角得到, 并有 $\max \left\{\left\|\boldsymbol{Q}_{i+1}^{r}-\boldsymbol{Q}_{i}^{r}\right\|\right\}_{i=0}^{M} \leqslant 2 \cdot \max \left\{\left\|\boldsymbol{P}_{i+1}^{r}-\boldsymbol{P}_{i}^{r}\right\|\right\}_{i=0}^{M-1}$. 同时 $\boldsymbol{\Gamma}_{1}$ 和 $\boldsymbol{\Gamma}_{2}$ 的距离 满足

$$
\operatorname{dist}\left(\boldsymbol{\Gamma}_{1}, \boldsymbol{\Gamma}_{2}\right)=\max \left\{\min \left\{\|\boldsymbol{P}-\boldsymbol{Q}\| \mid \boldsymbol{P} \in \boldsymbol{\Gamma}_{1}, \boldsymbol{Q} \in \boldsymbol{\Gamma}_{2}\right\}\right\} \leqslant \max \left\{\left\|\boldsymbol{P}_{i+1}^{r}-\boldsymbol{P}_{i}^{r}\right\|\right\}_{i=0}^{M-1} .
$$

如果对 $\hat{\boldsymbol{T}}$ 中每个 $\tau_{i}(i=0, \ldots, n)$ 都插入一个节点, 就得到新的控制多边形 $\boldsymbol{\Gamma}_{3}$. 我们有

$$
\operatorname{dist}\left(\boldsymbol{\Gamma}_{1}, \boldsymbol{\Gamma}_{3}\right) \leqslant\left(1+2+2^{2}+\cdots+2^{n}\right) \cdot \max \left\{\left\|\boldsymbol{P}_{i+1}^{r}-\boldsymbol{P}_{i}^{r}\right\|\right\}_{i=0}^{M-1}=\left(2^{n+1}-1\right) \cdot \max \left\{\left\|\boldsymbol{P}_{i+1}^{r}-\boldsymbol{P}_{i}^{r}\right\|\right\}_{i=0}^{M-1} .
$$

现在对每个节点 $\tau_{i}(i=0, \ldots, n)$ 都插入新节点直到 $r$ 重, 得到新的控制多边形 $\boldsymbol{\Gamma}_{4}$, 即有

$$
\operatorname{dist}\left(\boldsymbol{\Gamma}_{1}, \boldsymbol{\Gamma}_{4}\right) \leqslant 2^{(n+1) k} \cdot \max \left\{\left\|\boldsymbol{P}_{i+1}^{r}-\boldsymbol{P}_{i}^{r}\right\|\right\}_{i=0}^{M-1} .
$$

这样曲线 $\boldsymbol{P}(t)$ 就被分割成 $M$ 个 C-Bézier 曲线段. 当升的阶数足够高时, C-Bézier 的控制多边形将收 玫到曲线 [24]. 所以 $\exists N_{2}>0$, 当 $r>N_{2}, \operatorname{dist}\left(\boldsymbol{\Gamma}_{4}, \boldsymbol{P}(t)\right)<\varepsilon / 2$, 取 $N_{3}=\max \left\{N_{1}, N_{2}\right\}$. 所以, 当 $r>N_{3}$,

$$
\operatorname{dist}\left(\boldsymbol{\Gamma}_{1}, \boldsymbol{P}(t)\right)<2^{(n+1) k} \cdot \frac{\varepsilon}{2^{(n+1) k+1}}+\frac{\varepsilon}{2}=\varepsilon .
$$

\section{3 几何生成法}

基于上述的几何收玫性, 我们可以推出 C-B- 样条曲线与 Bézier 曲线一样的几何生成法 ${ }^{[16]}$. 一条 $k$ 阶 C-B- 样条曲线的几何生成算法描述如下: 
输入: 初始的控制顶点 $\left\{\boldsymbol{P}_{i}\right\}_{i=0}^{m}$ 和节点区间 $\boldsymbol{T}$, 以及需要升阶的次数 $s$.

输出: 一条由 $\left\{\boldsymbol{P}_{i}\right\}_{i=0}^{m}$ 和 $\boldsymbol{T}$ 定义的 $k$ 阶 C-B- 样条曲线.

For $d=1: s$;

\{

For $j=0: n-1$;

\{

1. 通过对 $t_{j}$ 插入一个节点得到 $\boldsymbol{T}^{j}$;

2. 计算所有的 $\left\{a_{i, k}^{j}\right\}(i=0, \ldots, m+j+1)$;

3. 计算新的控制顶点 $\left\{\boldsymbol{P}_{i}^{j}\right\}(i=0, \ldots, m+j+1)$;

4. $j=j+1$;

\}

升阶一次得到新的节点区间 $\boldsymbol{T}$ 和新的控制顶点 $\left\{\boldsymbol{P}_{i}\right\}$;

$k=k+1 ; d=d+1$

\}

举例 一条 C-B-样条曲线: 初始控制多边形 $\boldsymbol{P}_{0}^{0}=(15,0), \boldsymbol{P}_{1}^{0}=(0,15), \boldsymbol{P}_{2}^{0}=(20,35), \boldsymbol{P}_{3}^{0}=(40,15)$, $\boldsymbol{P}_{4}^{0}=(25,0)$, 节点区间 $\boldsymbol{T}=\{0,0,0,0,0.6,1,1,1,1\}$. 见图 5 .

\section{6 结论}

本文给出了 C-B-样条曲线的一个高效的生成算法. 这个算法是基于升阶算子的. 只要对控制多边 形持续的进行割角, 就能够生成一条 C-B-样条曲线. 这个算法有明显的几何意义, 更稳定的计算, 很适 合硬件的实现. 同时它对其他形式的 B- 样条也是成立的. 未来我们将致力于计算升阶算子 $\boldsymbol{A}$ 中的精 确系数并详细分析算法复杂度.

致谢 感谢莫群副教授对本文英文版的修改.

\section{参考文献}

1 Zhang J W. Two different forms of C-B-splines. Comput Aided Geom Des, 1997, 14: 31-41

2 Zhang J W. C-curves: an extension of cubic curves. Comput Aided Geom Des, 1996, 13: 199-217

3 Wang G Z, Chen Q Y, Zhou M H. NUAT B-spline curves. Comput Aided Geom Des, 2004, 21: 193-205

4 闵春燕, 汪国昭. C-B- 样条基是 B- 基. 浙江大学学报 (理学版), 2004, 31: 148-150

5 Mainar E, Peña J M, Sanchez-Reyes J. Shape preserving alternatives to the rational Bézier model. Comput Aided Geom Des, 2001, 18: 37-60

6 曾庭俊, 王卫民, 张纪文. C-B 样条旋转曲面造型研究. 工程图学学报, 2004, 25: 104-108

7 Yang Q M, Wang G Z. Inflection points and singularities on C-curves. Comput Aided Geom Des, 2004, 21: 207-213

8 Mainar E, Peña J M. A basis of C-Bézier splines with optimal properties. Comput Aided Geom Des, 2002, 19: 291-295

9 Hoffmann M, Juhasz I. On interpolation by spline curves with shape parameters. In: Proceedings of Geometric Modeling and Processing. Hangzhou: Zhejiang University, 2008. 205-214

10 Hoffmann M, Li Y J, Wang G Z. Paths of C-Bézier and C-B-spline curves. Comput Aided Geom Des, 2006, 23: 463-475

11 林兴, 罗国民, 张纪文. 基于 C-B 样条的 Catmull-Clark 细分曲面. 中国图象图形学报, 2002, 7: 876-881 
12 曾庭俊, 罗国民, 张纪文. Catmull-Clark 细分曲面的形状调整. 计算机辅助设计与图形学学报, 2004, 16: 707-711

13 曾庭俊. C-B 样条造型及其细分曲面系统的开发. 硕士学位论文. 杭州: 浙江大学, 2004

14 Wang W T, Wang G Z. Trigonometric polynomial B-splines with shape parameter. Prog Nat Sci, 2004, 14: 1023-1026

15 蒋春娟, 唐月红. 高阶 C-B 样条曲线曲面的显式形式. 高等学校计算数学学报, 2008, 30: 26-39

16 王国瑾, 汪国昭, 郑建民. 计算机辅助几何设计. 北京: 高等教育出版社, Heidelberg: Springer, 2001. 8-9

17 Huang Q X, Hu S M, Ralph R M. Fast degree elevation and knot insertion for B-spline curves. Comput Aided Geom Des, 2005, 22: 183-197

18 Liu W. A simple, efficient degree raising algorithm for B-spline curves. Comput Aided Geom Des, 1997, 14: 693-698

19 孙景楠, 王仁宏. B-样条曲线升阶算法中问题及其解决办法. 大连理工大学学报, 2003, 43: 397-398

20 De B C. Cutting corners always works. Comput Aided Geom Des, 1987, 4: 125-131

21 Paluszny M, Prautzsch H, Schäfer M. A geometric look at corner cutting. Comput Aided Geom Des, 1997, 14: 421-447

22 Wang G Z, Deng C Y. On the degree elevation of B-spline curves and corner cutting. Comput Aided Geom Des, 2007, 24: $90-98$

23 Chen Q Y, Wang G Z. A class of Bézier-like curves. Comput Aided Geom Des, 2003, 20: 29-39

24 Dong C S, Wang G Z. On convergence of the control polygon series of C-Bézier curves. In: Proceedings of Geometric Modeling and Processing. Beijing: Tsinghua University, 2004. 49-56 